\title{
Early Serum Heme Oxygenase-1, Soluble Vascular Endothelial Growth Factor Receptor-1, and B-Cell Lymphoma/Leukemia-2 Levels and Unfavorable Obstetric Outcomes
}

\author{
Havva SUTCU1, G. Fusun VAROL'1, Cihan INAN1, Isil UZUN'1, N. Cenk SAYIN1 \\ Edirne, Türkiye
}

\begin{abstract}
OBJECTIVE: To examine early pregnancy levels of serum heme oxygenase-1, soluble vascular endothelial growth factor receptor-1, B-cell lymphoma/leukemia-2 in relation to unfavorable pregnancy outcomes, including preeclampsia, fetal growth restriction, spontaneous preterm birth, gestational diabetes mellitus and fetal macrosomia.

STUDY DESIGN: A total of randomly selected 140 pregnancies were included in this prospective study. Peripheral blood samples were obtained between $11^{1 / 7}$ and $13^{6 / 7}$ gestational weeks. All pregnancies were followed up until the outcomes were obtained and classified as preeclampsia, fetal growth restriction, spontaneous preterm birth, gestational diabetes mellitus, fetal macrosomia, and uncomplicated ones.

RESULTS: Significantly high levels of early serum heme oxygenase-1 were found in the cases who subsequently developed preeclampsia, spontaneous preterm birth, and fetal macrosomia $(p<0.05)$, in concordance with high soluble vascular endothelial growth factor receptor-1 levels of the preeclampsia group, however, B-cell lymphoma/leukemia-2 s were similar in all groups. As soluble vascular endothelial growth factor receptor-1 predicted preeclampsia at a value of $11.905 \mathrm{ng} / \mathrm{mL}$, the cutoff values for the heme oxygenase-1 to predict preeclampsia, spontaneous preterm birth, and fetal macrosomia were $0.372 \mathrm{ng} / \mathrm{mL}, 0.354 \mathrm{ng} / \mathrm{mL}$ and $0.494 \mathrm{ng} / \mathrm{ml}$, respectively.
\end{abstract}

CONCLUSION: Elevated first trimester heme oxygenase-1 levels are remarkable in the pregnancies associated with preeclampsia, spontaneous preterm birth, and fetal macrosomia in comparison with uncomplicated ones.

Keywords: B-cell lymphoma/leukemia-2, Early pregnancy, Heme oxygenase-1, Pregnancy complications, Soluble vascular endothelial growth factor receptor-1

Gynecol Obstet Reprod Med 2022;28(2):122-128

1 Trakya University Faculty of Medicine Department of Obstetrics \& Gynecology Division of Perinatology, Edirne, Türkiye

Address of Correspondence: G. Fusun Varol

Trakya University, Faculty of Medicine,

Department of Obstetrics\&Gynecology,

Perinatology Division, 22030, Edirne, Türkiye

fgvarol@yahoo.com

Submitted for Publication: 22.12.2020 Revised for Publication: 18.02.2021 Accepted for Publication: 06.05.2021 Online Published: 20.05.2021

ORCID IDs of the authors:

GFV: 0000-0003-1918-4746,

IU: 0000-0003-3196-776X,

HS: 0000-0003-2028-340X,

CI: 0000-0002-4872-1689,

NCS: 0000-0001-5491-5431

\begin{tabular}{|c|c|}
\hline \multirow{3}{*}{ 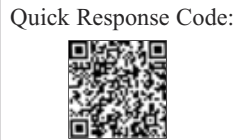 } & Access this article online \\
\hline & $\begin{array}{l}\text { Website: www.gorm.com.tr } \\
\text { e- mail: info@gorm.com.tr }\end{array}$ \\
\hline & DOI:10.21613/GORM.2021.1177 \\
\hline
\end{tabular}

How to cite this article: Sutcu H. Varol GF. Inan C. Uzun I. Sayin NC. Early Serum Heme Oxygenase-1, Soluble Vascular Endothelial Growth Factor Receptor-1, and B-Cell Lymphoma/Leukemia-2 Levels and Unfavorable Obstetric Outcomes. Gynecol Obstet Reprod Med. 2022;28(2):122-128

\section{Introduction}

Trophoblast-mediated spiral artery remodeling in early pregnancy is necessary for oxygen and nutrient supply by the placenta (1-3). An impaired balance between reactive oxygen species and antioxidants eventually leads to placental insufficiency (4). Nuclear factor erythroid 2-related factor (Nrf2)regulated heme oxygenase-1 (HO-1) exerts a cytoprotective effect against oxidative injury and regulates apoptosis and angiogenesis of the placenta (5). In addition, HO-1 attenuates the symptoms of preeclampsia (PE) (6). Furthermore, the transfection of human HO-1 in an animal study increased pup size; on the other hand, HO-1 deficiency caused fetal growth restriction (FGR) (7).

Defective placentation has been found to be associated with unfavorable obstetric outcomes such as PE, FGR, and 
preterm birth (8). The release of circulating maternal antiangiogenic factors during this defective placentation induces poor perfusion, hypoxia, and ischemia of the placenta. Soluble vascular endothelial growth factor receptor-1 (sVEGFR-1), an antiangiogenic factor, antagonizes the proangiogenic activity of vascular endothelial growth factor (VEGF) (9). Therefore, increased levels of sVEGFR-1 may play a central role in the pathogenesis of placental disorders, including PE and FGR $(10,11)$.

Trophoblast cell destiny appears to be regulated by diverse members of the B-cell lymphoma/leukemia-2 (Bcl-2) family. B-cell lymphoma/leukemia-2 (Bcl-2), an anti-apoptotic protein that regulates apoptosis by suppressing cell death, is a member of the Bcl-2 family. During early placentation, Bcl-2 levels are elevated when trophoblast apoptosis is minimal. Increased trophoblast cell proliferation and apoptosis are characteristic features of PE $(12,13)$.

In this preliminary randomized prospective study, the relationship between first-trimester serum HO-1 and Bcl-2 levels, which are protective against oxidative stress and apoptosis, and sVEGFR-1, which is a potent angiogenesis antagonist, and unfavorable obstetric outcomes, such as PE, FGR, spontaneous preterm birth (SPB), gestational diabetes mellitus (GDM), and fetal macrosomia (FM), were evaluated. Additionally, the possible cutoff values for these markers were defined. These biomarkers have clinical potential for predicting unfavorable obstetric outcomes.

\section{Material and Method}

This randomized prospective study was conducted at Trakya University, Faculty of Medicine, Department of Perinatology between January 2018 and February 2019. Informed consent was provided by all patients before enrollment in the study. Although 166 singleton pregnancies initially undergoing first-trimester screening were included in the study, 26 pregnancies were excluded later, as shown in the flowchart (Figure 1). The study ultimately continued with 140 patients.

Ethical approval was obtained from the Ethics Committee of Trakya University (TÜTF-BAEK No: 2018/60). All procedures were performed according to the Declaration of Helsinki.

\section{Study population}

Participants with smoking, alcohol consumption, multiple pregnancies, fetal chromosomal or structural anomalies, intrauterine or perinatal infections, antiphospholipid antibody syndrome or maternal connective tissue, cardiac, or renal diseases were excluded. Patients who had preterm premature rupture of the membranes (PPROM) or iatrogenic preterm labor were also excluded.

\section{Data source}

Peripheral blood samples were collected between $11^{0 / 7}$ and $13^{6 / 7}$ gestational weeks (GWs) for the first-trimester screening and they were centrifuged at 4000 revolutions per minute for 10 minutes. Afterward, $3 \mathrm{~mL}$ of residual samples were kept in Eppendorf tubes at $-80^{\circ} \mathrm{C}$ until assayed for the $\mathrm{HO}-1$, sVEGFR-1, and Bcl-2. All pregnancies were followed up until they were completed. Serum HO-1 (Shanghai YL Biotech Co., Ltd., China), sVEGFR-1 (Shanghai YL Biotech Co., Ltd., China), and Bcl-2 (Shanghai YL Biotech Co., Ltd., China) were measured by enzyme-linked immunosorbent assay (ELISA). The values for HO-1, sVEGFR-1, and Bcl-2 were adjusted for the covariates of GWs at sampling, maternal body mass index (BMI), and age. The final data set was analyzed in six groups according to the obstetric outcomes (PE, FGR, SPB, GDM, FM, and uncomplicated). Pregnancies complicated with both PE and/or FGR and GDM were excluded from the study (Figure 1).

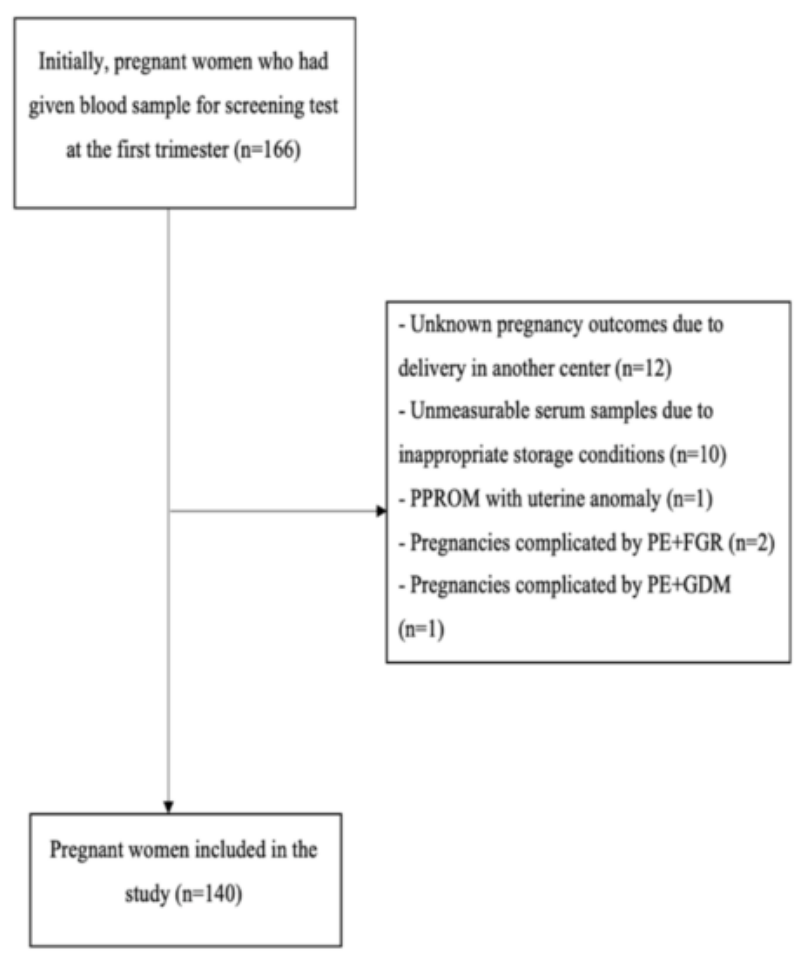

Figure 1: Flowchart of pregnant women in the study.

PE: Preeclampsia; GDM: Gestational diabetes mellitus; FGR: Fetal growth restriction; PPROM: Preterm premature rupture of membranes.

Preeclampsia is defined as hypertension and proteinuria after the $20^{\text {th }}$ week of gestation (14). FGR is defined as estimated fetal weight under the $10^{\text {th }}$ percentile and a pulsatility index of the umbilical artery higher than the $95^{\text {th }}$ percentile (15). SPB is defined as a birth before the $37^{\text {th }} \mathrm{GW}$ (16). GDM is diagnosed if one measurement is higher than the limit values for fasting ( $\geq 92 \mathrm{mg} / \mathrm{dL})$, the $1^{\text {st }}$ hour $(\geq 180 \mathrm{mg} / \mathrm{dL})$ or the 2nd hour ( $\geq 153 \mathrm{mg} / \mathrm{dL})$ with 75 -g oral glucose tolerance test between $24^{\text {th }}$ and $28^{\text {th }}$ GWs (17). FM is defined by estimated fetal weight above $4000 \mathrm{~g}$ (18). 


\section{Statistical analysis}

The Number Cruncher Statistical System 2007 (Kaysville, Utah, USA) was used for statistical analysis. The normally distributed quantitative data and demographic statistics such as mean, median, standard deviation, frequency, ratio, and minimum-maximum were tested by The Shapiro-Wilk test and graphics. The Kruskal-Wallis and Dunn-Bonferroni tests were used to compare more than two groups of quantitative not normally distributed variables. The Fisher-FreemanHalton exact test compared the qualitative data.

Maternal age, BMI, gestational age at the sampling were taken as covariates and generalized linear modeling was used to examine the effects of the groups on variables. Receiver operating characteristic (ROC) curve analysis was used for an appropriate cut-off value determination. The DeLong method was used to compare the area under the ROC curves. The value of $p<0 ., 05$ was considered statistically significant.

\section{Results}

This study was conducted with 140 pregnancies who were analyzed as follows: 10 PEs (7.1\%), 21 FGRs (15\%), 10 SPBs (7.1\%), 17 GDMs (12.1\%), 10 FMs (7.1\%); and 72 uncomplicated pregnancies as the controls $(51.4 \%)$. The characteristics of the population were shown in table I.

A generalized linear model was created for HO-1, sVEGFR-1, and Bcl-2. This model was also readjusted for maternal age, BMI, and GWs at the time of sampling as covariate variables. The levels of HO-1, sVEGFR-1 and Bcl-2 are shown in table I.

After Bonferroni-corrected bilateral evaluation, the sVEGFR-1 values of the PE group were found to be higher than those of the controls $(p=0.01)$. Furthermore, the HO- 1 values of the controls were found to be lower than those of the SPB, FM, and PE groups $(p=0.001 ; p=0.002 ; p=0.014$, respectively). However, Bcl-2 levels were not significantly different among the groups $(\mathrm{p}>0.05)$.

ROC analysis was performed for HO-1 and sVEGFR-1 to compare the groups (Table II, III; Figure 2, 3). The area under the curve (AUC) of HO-1 and sVEGFR-1 for the PE group was created using the Delong method. There was no statistically significant difference in the AUCs of HO-1 and sVEGFR-1 between the PEs and the controls (Table II).

The temporary cutoff points for sVEGFR-1 for PEs and the cutoff points for HO-1 for PEs, SPBs, and FMs were calculated (Table II, III). At a value of $11.905 \mathrm{ng} / \mathrm{mL}$, sVEGFR1 predicted PEs with a sensitivity of $71.43 \%$ and a specificity of $63.89 \%$. At a value of $0.372 \mathrm{ng} / \mathrm{mL}, \mathrm{HO}-1$ predicted PEs with a sensitivity of $71.43 \%$ and a specificity of $68.06 \%$. Further, at a value of $0.354 \mathrm{ng} / \mathrm{mL} \mathrm{HO}-1$ predicted SPBs with a sensitivity of $70 \%$ and a specificity of $66.67 \%$. On the other hand, HO-1, at a value of $0.494 \mathrm{ng} / \mathrm{m} \mathrm{mL}$, anticipated FMs with a sensitivity of $70 \%$ and a specificity of $80.56 \%$.

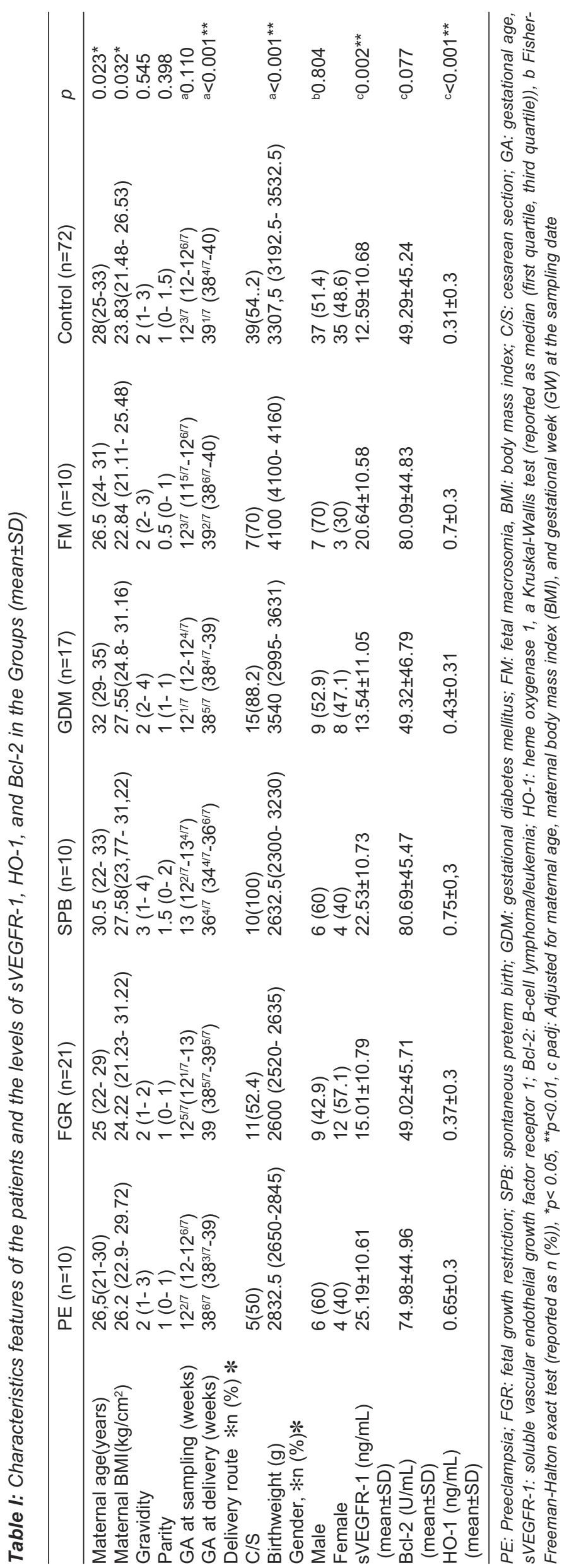


Table II: Cutoff values of the sVEGFR-1 and HO-1 for preeclampsia and under the ROC curves values

\begin{tabular}{lll}
\hline & sVEGFR-1 & HO-1 \\
\hline AuROC $(95 \% \mathrm{Cl})$ & $0.711(0.598-0.808)$ & $0.764(0.655-0.852)$ \\
p & $0.018^{*}$ & $<0.001^{* *}$ \\
Cutoff & $>11.905$ & $>0.372$ \\
Sensitivity $(95 \% \mathrm{Cl})$ & $71.43(29.0-96.3)$ & $71.43(29.0-96.3)$ \\
Specificity $(95 \% \mathrm{Cl})$ & $63.89(51.7-74.9)$ & $68.06(56.0-78.6)$ \\
PPV $(95 \% \mathrm{Cl})$ & $16.1(9.9-25.2)$ & $17.9(10.9-27.9)$ \\
NPV $(95 \% \mathrm{Cl})$ & $95.8(87.6-98.7)$ & $96.1(88.3-98.8)$ \\
\hline
\end{tabular}

${ }^{d} p$

AUC comparison

0.206

AUC: area under the ROC curves (95\% confidence interval); HO-1: heme oxygenase 1; NPV: negative predictive value; PPV: positive predictive value; sVEGFR-1: soluble vascular endothelial growth factor receptor 1, $d$ DeLong method, ${ }^{* *} p<0.01$

Table III: Cutoff values of HO-1 for adverse pregnancy outcomes and under the ROC curves values

\begin{tabular}{lll}
\hline HO-1 & SPB versus Control & FM versus Control \\
\hline AuROC $(95 \% \mathrm{Cl})$ & $0.767(0.661-0.854)$ & $0.793(0.689-0.875)$ \\
$\mathrm{p}$ & $0.003^{* *}$ & $0.001^{* *}$ \\
Cut-off & $>0.354$ & $>0.494$ \\
Sensitivity $(95 \% \mathrm{Cl})$ & $70(34.8-93.3)$ & $70(34.8-93.3)$ \\
Specificity $(95 \% \mathrm{Cl})$ & $66.67(54.6-77.3)$ & $80.56(69.5-88.9)$ \\
PPV $(95 \% \mathrm{Cl})$ & $22.6(9.6-41.1)$ & $33.3(14.6-57)$ \\
NPV $(95 \% \mathrm{Cl})$ & $94.1(83.8-98.8)$ & $95.1(86.3-99)$
\end{tabular}

SPB: spontaneous preterm birth; FM: fetal macrosomia, AUC: area under the ROC curves (95\% confidence interval); HO-1: heme oxygenase 1; NPV: negative predictive value; PPV: positive predictive value, ${ }^{* *} p<0.01$

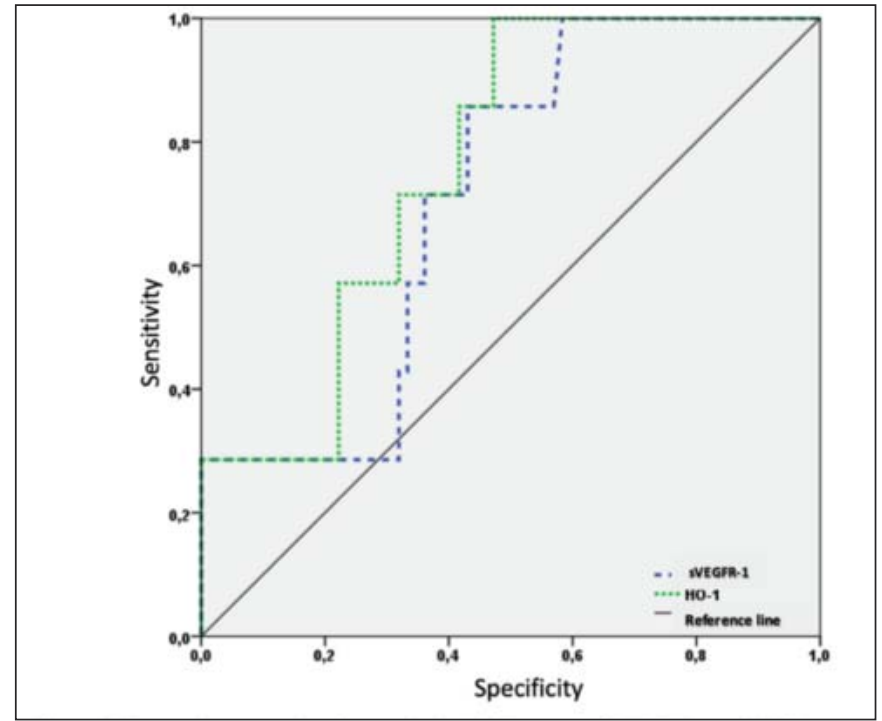

Figure 2: The receiver operating characteristic (ROC) curves of sVEGFR-1 and HO-1 to differentiate preeclampsia from controls.

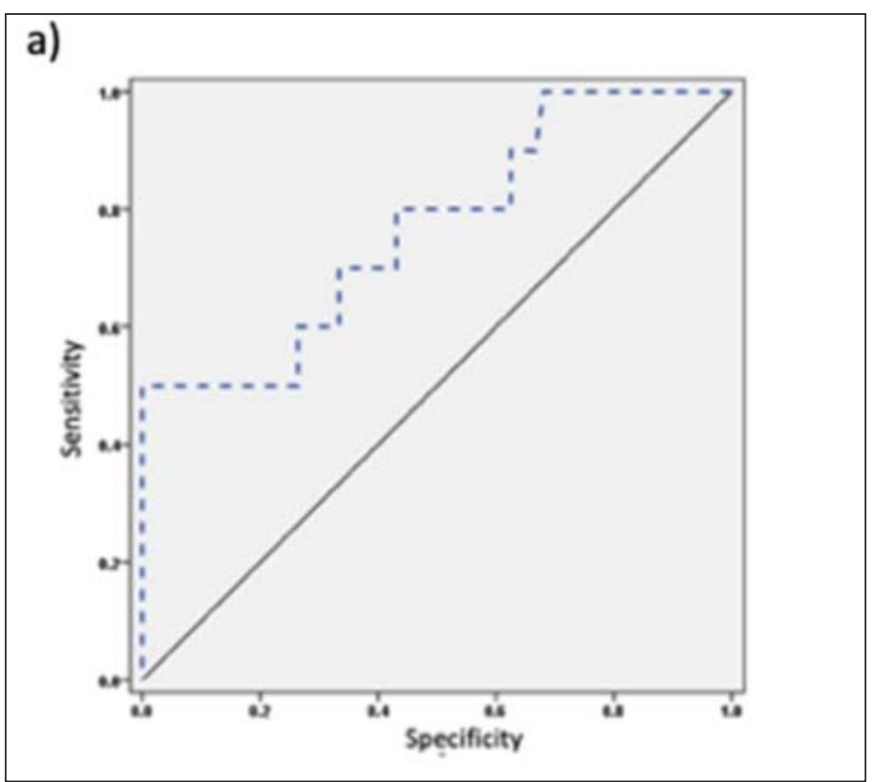

Figure 3a: The receiver operating characteristic curve of $\mathrm{HO}-$ 1 to differentiate spontaneous preterm birth from Controls 


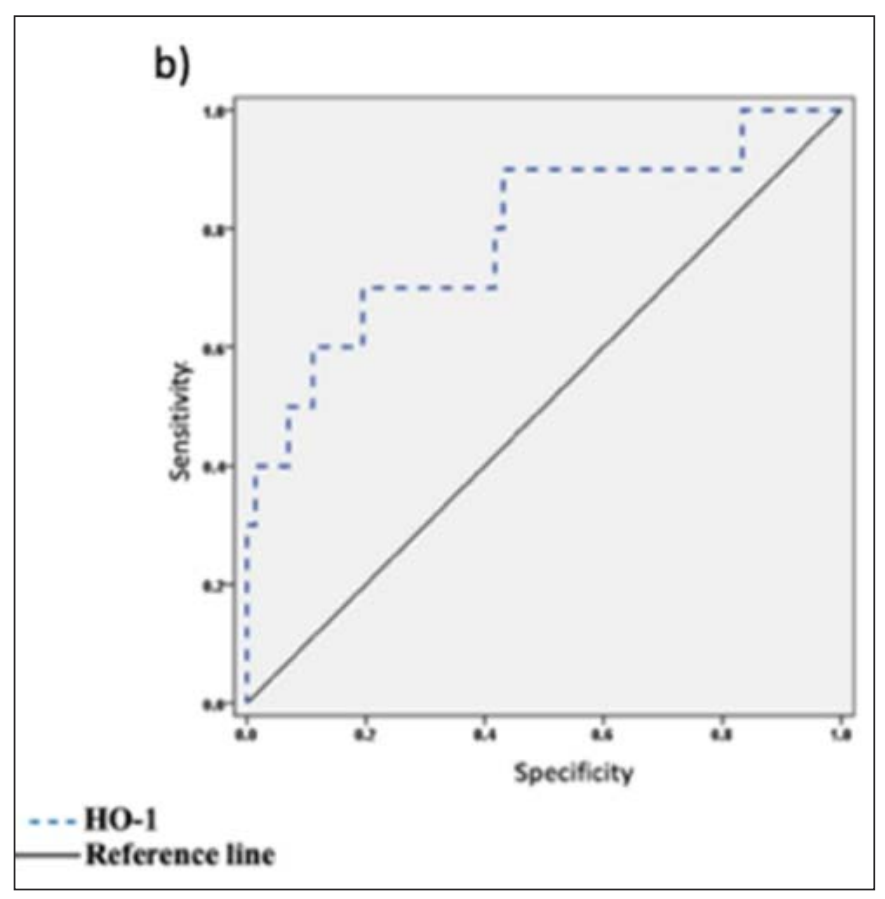

Figure 3b:The receiver operating characteristic curve of $\mathrm{HO}-1$ to differentiate fetal macrosomia from Controls

\section{Discussion}

HO-1 is considered to be a marker of increased oxidative stress in cultured trophoblasts (19). The induction of HO-1 in trophoblasts maintains pregnancy in humans. However, reduced HO-1 levels result in pregnancies with PE or FGR (20). These stressful conditions induce the multifunctional regulator nuclear factor erythroid 2-related factor ( $\mathrm{Nrf2}$ ) pathway, which increases the transcription of Nrf2-regulated genes such as $\mathrm{HO}-1$, so this response is considered not only cytoprotective but also a modulator of pregnancy longevity. HO-1 provides beneficial effects against oxidative injury, apoptosis, and angiogenesis (6).

While some studies have found high serum HO-1s in severe PE (21), others have reported decreased expression of HO-1 mRNA (22). Vitoratos et al. studied 31 pregnant women divided into severe PE, mild PE, and control groups. Serum levels of HO-1 were measured in the third trimester. The severe PE group had significantly higher serum HO-1 levels than the mild PE and control groups (21). Nakamura et al. found divergent results in their study involving 24 pregnant women with PE and 24 normotensive pregnant women in the third trimester. They found that the mRNA expression of HO1 decreased in the cellular component of the blood in patients with PE (22). Farina et al. followed 30 pregnant women who underwent chorionic villous sampling (CVS) at $11^{\text {th }}$ GWs. Five of them developed PE later in gestation, and the mRNA expression of HO-1 was significantly lower in the CVS tissues from patients with PE (23).

In our PE group, the first trimester HO-1 levels were found to be higher than those in the controls. The higher levels of HO-1 in PE may be due to the activation of the Nrf2/HO-1 pathway in the first trimester as a response to enhanced oxidative stress. We also determined that the cutoff level of HO-1 in the first trimester was $0.372 \mathrm{ng} / \mathrm{mL}$ to predict PE. This cutoff level may be useful in clinical practice to predict pregnancies at high risk of $\mathrm{PE}$.

An imbalance between oxidants and antioxidants has been reported in the etiology of SPB, but it is not clear whether oxidative stress is an etiology or a result of SPB (24). There are no human-origin articles in the literature investigating the relationship between HO-1 and SPB. In a mouse model study, it was found that statins could prevent PB by increasing the synthesis, expression, and activity of HO-1 in the myometrium and cervix (25). However, the current study showed that the first-trimester serum HO-1 was significantly higher in the SPB group. Our cutoff value was $0.354 \mathrm{ng} / \mathrm{mL}$ to predict SPB in the current pregnancy. To the best of our knowledge, there is no first-trimester cutoff value mentioned for HO-1 for the prediction of SPB and PE in the previous literature.

The modulatory effect of HO-1 on placental growth factors is also important in fetal growth (26). High FM-associated levels of HO-1 again reveal the role of HO-1 in fetal growth and the maintenance of pregnancy. The birth weights were between 2530 and $2635 \mathrm{~g}$ in our FGR group. However, if there were more severe FGR cases in our study, the significant results in terms of the serum markers studied may be observed.

There are contradictory results about an association of HO-1 with GDM in the literature. Low maternal HO-1 levels of approximately $16^{\text {th }} \mathrm{GW}$ have been reported in those who develop GDM later on. (27). On the other hand, the expression of HO-1 mRNA has been found to be elevated in mononuclear cells of GDM cases at approximately $24^{\text {th }}$ GW (28). The mechanism explaining the association of GDM with HO-1 in the first trimester is unclear (29). Since our serum samples were obtained in the first trimester, we could not come to any firm conclusions about HO-1 levels and GDM.

Oxidative stress in trophoblasts may modulate the production of antiangiogenic factors through the upregulation of sVEGFR-1 (19). The sVEGFR-1 levels studied at $12^{\text {th }}-16^{\text {th }}$ GWs were high in one study, but no cutoff value was given (30). Another study found high sVEGFR-1 mRNA in the chorionic villous samples among patients who developed $\mathrm{PE}$ later on (23). The first-trimester serum sVEGFR-1 levels in the PE group were found to be higher in our study.

Several studies have examined PE in the third trimester. In one study, the maternal sVEGFR-1 was not different between late PE and controls; however, the sVEGFR-1 of early-onset PE was found to be significantly higher in the third trimester (31). In our study, we found a sVEGFR-1 cutoff value of $11.905 \mathrm{ng} / \mathrm{mL}$ for the PE group. Unlike the studies in the ad- 
vanced weeks, we took blood samples in the first trimester before PE developed. This cutoff value may be employed to identify pregnancies likely to develop PE. There are also several studies demonstrating a relationship between sVEGFR-1 and FGR $(11,32)$. The reason for our nonsignificant results may be that the average birth weights in our FGR group were not all that low.

$\mathrm{Bcl}-2$ is an anti-apoptotic protein that regulates apoptosis. In our previous study, we found significantly higher serum Bcl-2 levels in the mild PE group than in women who had severe PE. (33). In contrast, some studies have reported that Bcl2 levels are unchanged in PE $(34,35)$. In our study, there were no significant differences in the Bcl-2 levels in the first trimester in all groups. Perhaps the protective effects of Bcl-2 might be more effective in advanced gestational weeks. Therefore, we might have found a difference if the serum samples were taken from our patients after $14^{\text {th }} \mathrm{GWs}$.

To the best of our knowledge, this is the first study to investigate serum HO-1, Bcl-2, and sVEGFR-1 levels in the first trimester together for the prediction of unfavorable obstetric outcomes. The prospective study design of our study allowed us to follow patients from the first trimester until the end of pregnancy. Thus, unfavorable obstetric outcomes were determined, and the study groups were formed properly. Regarding the limitations of our study, increasing our sample size would help to obtain more meaningful results.

In conclusion, in the first trimester of pregnancy, high serum sVEGFR-1 levels are associated with the development of PE, while high HO-1 levels are associated with PE, SPB, and FM. This may show that protective mechanisms such as the Nrf2 pathway are activated in response to oxidative stress in the placenta during the first trimester.

Acknowledgments: We like to thank "Research Foundation of the Trakya University" for the funding support.

Conflict of interest: The authors declare no conflict of interest. Funding: This study was supported by the Research Fund of the Trakya University (Project number 2018/212).

Authors' contributions: Havva Sutcu, G. Fusun Varol raised the presented idea. Havva Sutcu, G. Fusun Varol designed the study. Havva Sutcu and Cihan Inan conducted the analyses. G. Fusun Varol, Cihan Inan, Isil Uzun, N. Cenk Sayin developed the first draft of the manuscript. All authors contributed to the writing of the paper, and have read and approved the final manuscript. All authors read and approved the final manuscript.

\section{References}

1. Burton GJ, Jauniaux E, Watson AL. Maternal arterial connections to the placental intervillous space during the first trimester of human pregnancy: The Boyd collection revisited. Am J Obstet Gynecol. 1999;181(3):718-24. Doi: 10.1016/s0002-9378(99)70518-1.
2. Rodesch F, Simon P, Donner C, Jauniaux E. Oxygen measurements in endometrial and trophoblastic tissues during early pregnancy. Obstet Gynecol. 1992;80(2):283-5. PMID: 1635745

3. Haram K, Mortensen JH, Myking O, Roald B, Magann EF, Morrison JC. Early development of the human placenta and pregnancy complications. J Matern Fetal Neonatal Med. 2020;33(20):3538-45. Doi:10.1080/ 14767058.2019.1578745.

4. Schoots MH, Gordijn SJ, Scherjon SA, van Goor H, Hillebrands JL. Oxidative stress in placental pathology. Placenta. 2018;69:153-61. Doi: 10.1016/j.placenta.2018. 03.003 .

5. Boyd JD, Hamilton WJ. The human placenta. Cambridge (MA): W. Heffer\&Sons;1970.

6. Loboda A, Damulewicz M, Pyza E, Jozkowicz A, Dulak J. Role of Nrf2/HO-1 system in development, oxidative stress response and diseases: an evolutionarily conserved mechanism. Cell Mol Life Sci. 2016;73(17):3221-47. Doi: 10.1007/s00018-016-2223-0.

7. Kreiser D, Nguyen X, Wong R, Seidman D, Stevenson D, Quan S, et al. Heme oxygenase-1 Modulates Fetal Growth in the Rat. Lab Invest. 2002;82(6):687-92. Doi: 10.1097/01.lab.0000017167.26718.f2.

8. Brosens I, Pijnenborg R, Vercruysse L, Romero R. The 'Great Obstetrical Syndromes' are associated with disorders of deep placentation. Am J Obstet Gynecol. 2011;204(3):193-201. Doi: 10.1016/j.ajog.2010.08.009.

9. Clark DE, Smith SK, Sharkey AM, Charnock-Jones DS. Localization of VEGF and expression of its receptors flt and KDR in human placenta throughout pregnancy. Hum Reprod. 1996;11(5):1090-8. Doi: 10.1093/oxfordjournals.humrep.a019303.

10. Mckeeman GC, Ardill JE, Caldwell CM, Hunter AJ, Mc Clure N. Soluble vascular endothelial growth factor reseptör-1(sFlt-1) is increased throughout gestation in patients who have preeclampsia develop. Am J Obstet Gynecol. 2004;191(4):1240-6. Doi:10.1016/j.ajog.2004. 03.004.

11. Birdir C, Droste L, Fox L, Frank M, Fryze J, Enekwe A, et al. Predictive value of sFlt-1, PlGF, sFlt-1/PlGF ratio and PAPP-A for late-onset preeclampsia and IUGR between 32 and 37 weeks of pregnancy. Pregnancy Hypertens. 2018;12:124-8. Doi: 10.1016/j.preghy.2018. 04.010.

12. Huppertz B, Frank HG, Kingdom JC, Reister F, Kaufmann P. Villous cytotrophoblast regulation of the syncytial apoptotic cascade in the human placenta. Histochem Cell Biol. 1998;110(5):495-508. Doi: 10.1007/s004180050311.

13. Ray J, Jurisicova A, Caniggia I. IFPA trophoblast research award lecture: The dynamic role of Bcl-2 family members in trophoblast cell fate. Placenta. 2009;30:96-100. Doi: 10.1016/j.placenta. 2001.11.008.

14. Brown MA, Magee LA, Kenny LC, Karumanchi SA, McCarthy FP, Saito S, et al. The hypertensive disorders of 
pregnancy: ISSHP classification, diagnosis \& management recommendations for international practice. Pregnancy Hypertens. 2018;13:291-310. Doi: 10.1016/j. preghy.2018.05.004.

15. Duan J, Chabot-Lecoanet AC, Perdriollle-Galet E, Christov C, Hossu G, Cherifi A, et al. Utero-placental vascularization in normal and preeclamptic and intra-uterine growth restriction pregnancies: third trimester quantification using 3D power Doppler with comparison to placental vascular morphology (EVUPA): a prospective controlled study. BMJ Open. 2016;6(e):e009909. Doi: 10. 1136/bmjopen-2015-009909.

16. Goldenberg RL, Culhane JF, Iams JD, Romero R. Epidemiology and causes of preterm birth. Lancet. 2008;371 (9606):75-84. Doi: 10.1016/S0140-6736(08) 60074-4.

17. Lapolla A, Dalfrà MG, Ragazzi E, De Cata AP, Fedele D. New International Association of the Diabetes and Pregnancy Study Groups (IADPSG) recommendations for diagnosing gestational diabetes compared with former criteria: a retrospective study on pregnancy outcome. Diabet Med. 2011;28(9):1074-7. Doi: 10.1111/j.1464-5491.2011. 03351.x.

18. Chauhan SP, Grobman WA, Gherman RA, Chauhan VB, Chang G, Magann EF, et al. Suspicion and treatment of the macrosomic fetus: a review. Am J Obstet and Gynecol. 2005;193(2):332-46. Doi: 10.1016/j.ajog.2004. 12.020.

19. Li H, Gu B, Zhang Y, Lewis DF, Wang Y. Hypoxia-induced increase in soluble Flt-1 production correlates with enhanced oxidative stress in trophoblast cells from the human placenta. Placenta. 2005;26(2-3):210-7. Doi:10. 1016/j.placenta.2004.05.004.

20. Zenclussen ML, Linzke N, Schumacher A, Fest S, Meyer $\mathrm{N}$, Casalis PA, et al. Heme oxygenase-1 is critically involved in placentation, spiral artery remodeling, and blood pressure regulation during murine pregnancy. Front Pharmacol. 2014;5:291. Doi: 10.3389/fphar.2014.00291.

21. Vitoratos N, Papakonstantinou K, Deliveliotou A, Economou E, Panoulis C, Hassiakos D, et al. Antepartum and postpartum serum heme oxygenase-1 levels in preeclamptic and normotensive pregnant women. In Vivo. 2011;25(3):445-50. PMID: 21576421

22. Nakamura M, Sekizawa A, Purwosunu Y, Okazaki S, Farina A, Wibow N, et al. Cellular mRNA expressions of anti-oxidant factors in the blood of preeclamptic women. Prenatal Diagn. 2009;29(7):691-6. Doi: 10.1002/pd.2278.

23. Farina A, Zucchini C, De Sanctis P, Morano D, Sekizawa A, Purwosunu Y, et al. Gene expression in chorionic villous samples at 11 weeks of gestation in women who develop pre-eclampsia later in pregnancy: implications for screening. Prenat Diagn. 2011;31(2):181-5. Doi: 10.1002/ pd.2675.

24. Moore TA, Ahmad IM, Zimmerman MC. Oxidative Stress and Preterm Birth: An Integrative Review. Biol Res Nurs.

\section{8;20(5):497-512. Doi: 10.1177/1099800418791028.}

25. Gonzalez JM, Pedroni SM, Girardi G. Statins prevent cervical remodeling, myometrial contractions and preterm labor through a mechanism that involves hemoxygenase1 and complement inhibition. Mol Hum Reprod. 2014;20 (6):579-89. Doi: 10.1093/molehr/gau019.

26. Kreiser D, Nyugen X, Wong R, Seidman D, Stevenson D, Quan S, et al. Heme Oxygenase-1 modulates fetal growth in the rat. Lab Invest. 2002;82(6):687-92. Doi: 10.1097/ 01.lab. 0000017167.26718.f2.

27. Qiu C, Hevner K, Enquobahrie DA, Williams MA. Maternal serum Heme-Oxygenase-1 (HO-1) concentrations in early pregnancy and subsequent risk of gestational diabetes mellitus. PLoS One. 2012;7(11):e48060. Doi: 10. 1371/journal.pone.0048060.

28. Xin G, Du J, Wang YT, Liang TT. Effect of oxidative stress on heme oxygenase-1 expression in patients with gestational diabetes mellitus. Exp Ther Med. 2014; 7(2): 478-82. Doi: 10.3892/etm.2013.1435.

29. Plows JF, Stanley JL, Baker PN, Reynolds CM, Vickers MH. The pathophysiology of gestational diabetes mellitus. Int J Mol Sci. 2018;19(11):3342. Doi: 10.3390/ ijms 19113342.

30. Bian Z, Shixia C, Duan T. First trimester maternal serum levels of sFLT1, PGF and ADMA predict preeclampsia. PLoS One. 2015;10(4):e0124684. Doi: 10.1371/journal. pone. 0124684 .

31. Aldika Akbar MI, Herdiyantini M, Aryananda RA, Clninta N, Wardhana MP, Gumilar KE, et al. Serum heme oxygenase 1 (HO-1), soluble FM like tyrosine kinase (sFlt-1) level, and neonatal outcome in early onset, late onset preeclampsia, and normal pregnancy. Hypertens Pregnancy. 2018;37(4):175-81. Doi: 10.1080/10641955. 2018.1494187.

32. Vogtmann R, Kühnel E, Dicke N, Verkaik-Schakel RN, Plösch T, Schorle H, et al. Human sFLT1 leads to severe changes in placental differentiation and vascularization in a transgenic hsFLT1/rtTA FGR mouse model. Front Endocrinol (Lausanne). 2019;21:165. Doi: 10.3389/ fendo.2019.00165.

33. Varol F, Uzunoğlu R, Erbaş H, Süt N, Sayın C. VEGFR$1, \mathrm{Bcl}-2$, and HO-1 ratios in pregnant women with hypertension. Clin Appl Thromb Hemost. 2015;21(3):285-8. Doi: $10.1177 / 1076029613498816$.

34. Allaire AD, Ballenger KA, Wells SR, McMahon MJ, Lessey BA. Placental apoptosis in preeclampsia. Obstet Gynecol. 2000;96(2):271-6. Doi: 10.1016/s00297844(00)00895-4.

35. Levy R, Smith SD, Yusuf K, Huettner PC, Kraus FT, Sadovsky Y, et al. Trophoblast apoptosis from pregnancies complicated by fetal growth restriction is associated with enhanced p53 expression. Am J Obstet Gynecol. 2002;186(5):1056-61. Doi: 10.1067/mob.2002.122250. 